\title{
RISKS AND CONSEQUENCES OF USING THE TRANSPORTAL TECHNIQUE IN RECONSTRUCTING THE ANTERIOR CRUCIATE LIGAMENT: RELATIONSHIPS BETWEEN THE FEMORAL TUNNEL, LATERAL SUPERIOR GENICULAR ARTERY AND LATERAL EPICONDYLE OF THE FEMORAL CONDYLE
}

Diego Costa Astur ${ }^{1}$, Vinicius Aleluia², Ciro Veronese Santos², Gustavo Gonçalves Arliani', Ricardo Badra², Saulo Gomes Oliveira², Camila Cohen Kaleka ${ }^{3}$, Moisés Cohen ${ }^{4}$

\section{ABSTRACT}

Objective: Define a security zone to avoid possibles vascular and ligamentar complications during anterior cruciate ligament reconstruction. Methods: Arthroscopic reconstruction using the transtibial and transportal technique in cadaver knees was performed followed by dissection and measurement of the distance between the femoral tunnel and the proximal attachment of the lateral collateral ligament and the femoral tunnel and the lateral superior genicular artery. Results: The measure of the analysed distances show us an aproximation between the major branch of the lateral superior genicular artery and the femoral insertion of the colateral lateral ligament and the femoral tunnel during the transportal technique. Conclusion: We realize that the use of technical ship it to arthroscopic ACL reconstruction has a higher probability of injury to the lateral geniculate artery and insertion of the lateral collateral ligament, promoting post-surgical complications such as instability of the knee, osteonecrosis of the femoral condyle and ligamentização graft.

Keywords - Anterior Cruciate Ligament; Surgical Procedures, Minimally Invasive; Arteries; Femur

\section{INTRODUCTION}

The anterior cruciate ligament (ACL) is one of the main structures of the knee and is responsible for anteroposterior and rotational stabilization ${ }^{(1)}$. It proximal insertion is in the medial surface of the lateral femoral condyle and its distal insertion is in the anterolateral depression of the intercondylar fossa of the tibia ${ }^{(1)}$ and is composed of two band: the anteromedial band and the posterolateral band ${ }^{(2,3)}$. Recreational or professional sports activity requires good knee function, and greater participation in sports among the general population exposes these individuals to increased risk of injury, and ACL lesions are very common. Surgical treatment is usually considered to be the best thera- peutic option for knees with a deficient ACL, and this results in approximately 100,000 reconstructions per year in the United States ${ }^{(4)}$.

Clinical and biomechanical studies have provided better understanding of this ligament in relation to joint dynamics and have contributed towards improvements in reconstruction operations seen over recent years. Arthroscopic reconstruction of the ACL is a commonly performed and successful orthopedic surgical procedure, and there is considerable variety in the techniques and materials used. The objective of surgical treatment is to reestablish knee stability, enable a return to sports activity and, over the long term, avoid joint osteoarthrosis ${ }^{(5,6)}$.

1 - Orthopedist at the Sports Traumatology Center, UNIFESP/EPM, São Paulo, SP, Brazil.

2 - Orthopedist; training to specialize in Knee Surgery at the Cohen Institute, São Paulo, SP, Brazil.

3 - Orthopedist at the School of Medical Sciences, Santa Casa de Misericórdia de São Paulo, São Paulo, SP, Brazil.

4 - Full Professor and Head of the Department of Orthopedics and Traumatology, UNIFESP/EPM, São Paulo, SP, Brazil.

Work performed in the Department of Orthopedics and Traumatology, Paulista School of Medicine, Federal University of São Paulo.

Correspondence: Rua Borges Lagoa 783, 5 andar, Vila Clementino, 04038-032 São Paulo, SP. E-mail: mcastur@yahoo.com

Work received for publication: October 26, 2011; accepted for publication: November 29, 2011.

The authors declare that there was no conflict of interest in conducting this work 
Among the techniques used for ACL reconstruction, the one most commonly performed is the transtibial technique, in which the femoral tunnel is produced using a guide introduced via the tibial tunnel, thus resulting in a verticalized and isometric bit non-anatomical position for the neoligament ${ }^{(7)}$. Studies have shown that there is great difficulty in achieving a horizontalized position for the femoral tunnel ${ }^{(8,9)}$ by means of the transtibial technique. Non-anatomical reconstruction of the ligament is considered to be the cause of some poor results from this surgery, such as persistence of rotational instability, which has stimulated development of techniques that would favor anatomical positioning for the neoligament ${ }^{(10)}$. The option of reconstructing both bands (anteromedial and posterolateral) has been shown to be effective ${ }^{(10-13)}$, but frequent technical difficulties, longer duration of the operation and greater cost of the fixation materials have been among the limitations on popularization of this technique.

Performing reconstruction by means of a transportal technique has been shown to be an option when it is possible to combine anatomical reproduction of the ligament (by means of independent positioning of the femoral and tibial tunnels) with the practicality of single-band reconstruction of the knee ${ }^{(14)}$. The neoligament results in a horizontalized position, thus providing greater anteroposterior and rotational stability $^{(15,16)}$ and biomechanical performance ${ }^{(16-18)}$, and minimizing occurrences of future osteoarthrosis ${ }^{(5,6)}$.

With a horizontalized and distal position for the femoral tunnel, its entry point is located at the center of the femoral footprint, at the midpoint between the insertions of the two bands in the ACL or at the site of the posterolateral band of the native ligament ${ }^{(19)}$. Horizontalization of the femoral tunnel resulting from the transportal anatomical technique ${ }^{(20)}$ exposes the guidewire of the femoral tunnel to an anatomical region of the lateral femoral condyle that is usually not approached $^{(21)}$. This region contains the main artery feeding the lateral femoral condyle and one of the main vascular branches of the popliteal artery: the lateral superior genicular artery ${ }^{(22,23)}$. Furthermore, the lateral epicondyle of the femur is close to this region, and this is the site of proximal insertion of the lateral collateral ligament and the tendon of the popliteal muscle. The risk of injury to the structures close to the lateral cortex of the femur may lead to significant complications $^{(24)}$.
The aims of the present study were to measure and compare the mean distance between the center of the tunnel in the lateral femoral condyle and the lateral epicondyle and main branch of the lateral superior genicular artery, while performing ACL reconstruction in cadaver knees by means of the transtibial and transportal techniques, and to be able to define the risk of injury to the structures studies and the possible consequences of such risks.

\section{METHODS}

This project was analyzed and approved by the Ethics Committee of the Federal University of São Paulo.

We used three knees from cadavers for studying the lateral femoral condyle, and these knees were presented with the distal $20 \mathrm{~cm}$ of the femur and the proximal $20 \mathrm{~cm}$ of the tibia. Firstly, all the arteries present at the extremities were identified and ligated using cotton thread, so that only the proximal and distal regions of the popliteal artery (the only one without an obstructed channel) would function as an entry and exit point for the contrast applied to the knees.

We introduced injections of non-transparent liquid silicone contrast in association with red stain through the popliteal artery in order to identify and measure the structures under examination. We performed arthroscopy on the knees in an appropriate laboratory using appropriate instruments. The entire ACL was damaged and resected, and then we introduced guidewires into the femur using the transportal and transtibial technique, thus simulating the exit point of the tunnels that are generally used (Figures 1 and 2).

The third phase of the study consisted of dissection of the joints. With the transtibial and transportal guidewires still in place, a lateral route was constructed and dissected down to the deeper layers of the posterolateral compartment, in order to view the entire length of the lateral superior genicular artery, from its start in the popliteal artery and the definition of the lateral epicondyle and structures inserted there (Figure 3).

The distance from the femoral tunnel to the proximal insertion of the lateral collateral ligament and the distance from the femoral tunnel to the main branch of the lateral superior genicular artery were measured and compared between the different techniques for ACL reconstruction that were examined. 


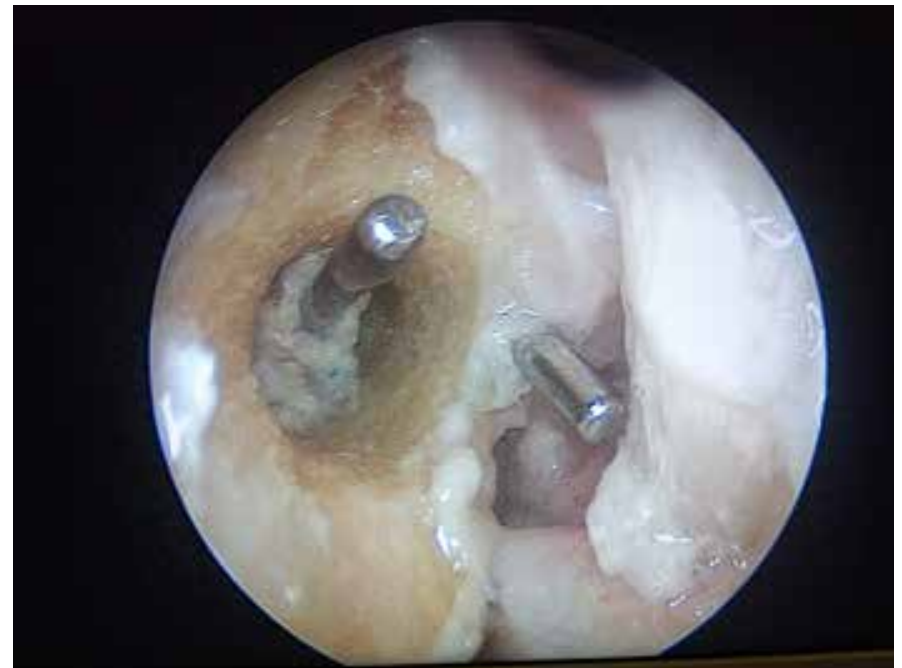

Figure 1 - Arthroscopic views of the femoral tunnel through using the transportal technique and the transtibial technique.

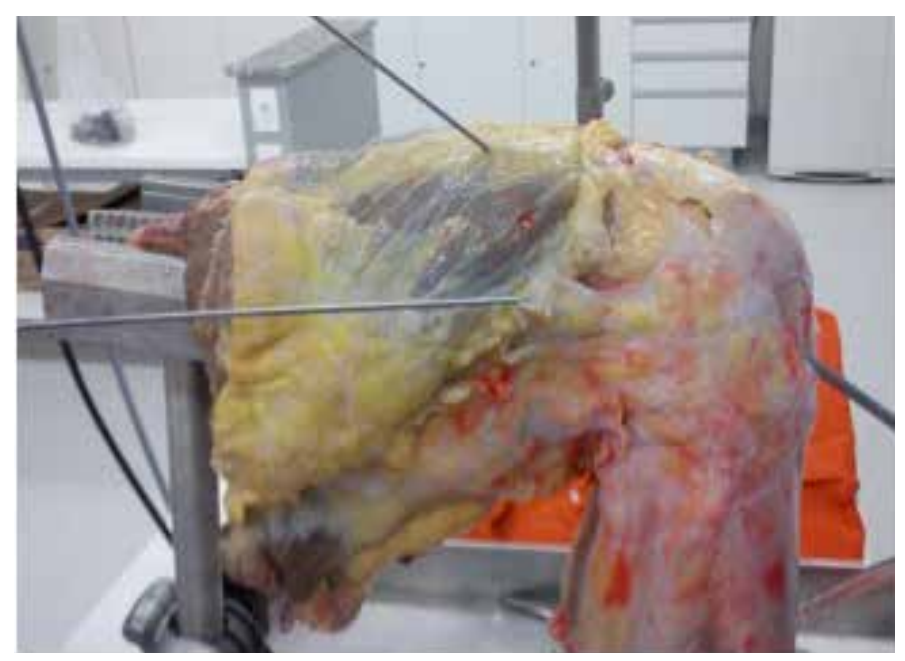

Figure 2 - Image of the cadaver knee showing injection of contrast after arthroscopy and placement of guidewires using the transtibial and transportal techniques before posterolateral dissection of the knee.

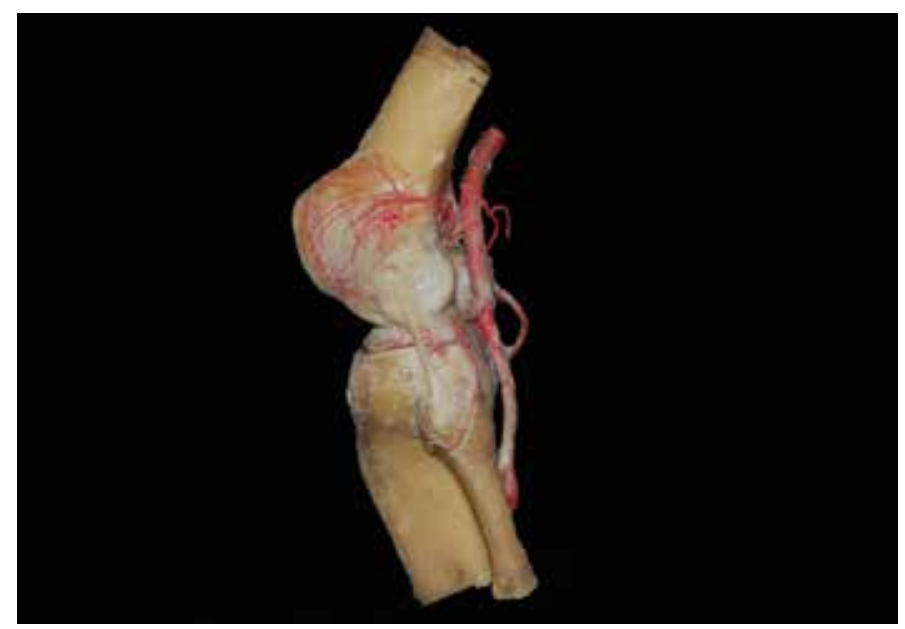

Figure 3 - Lateral view of the dissected knee after application of contrast through the femoral artery to measure the distance between the femoral tunnel and the structures of the lateral femoral condyle: the lateral superior genicular artery and the proximal insertion of the lateral collateral ligament.

\section{RESULTS}

The measurements of the distances analyzed showed that the main branch of the lateral superior genicular artery was brought closer to the proximal insertion of the lateral collateral ligament when the femoral tunnel was produced using the transportal technique.

The transtibial tunnel was on average $0.6 \mathrm{~cm}$ further away from the artery than the transportal tunnel and $2.2 \mathrm{~cm}$ further away from the proximal ligament insertion than the transtibial tunnel. All these values can be evaluated in Table 1.

Table 1 - Data obtained from measuring from the femoral tunnel to the lateral collateral ligament and to the main branch of the lateral superior genicular artery using the transtibial (TT) and transportal (TP) ligament reconstruction techniques on three different knees.

\begin{tabular}{c|c|c|c|c}
\hline & $\begin{array}{c}\text { TT tunnel- } \\
\text { vessel }\end{array}$ & $\begin{array}{c}\text { TT tunnel- } \\
\text { ligament }\end{array}$ & $\begin{array}{c}\text { TP tunnel- } \\
\text { vessel }\end{array}$ & $\begin{array}{c}\text { TP tunnel- } \\
\text { ligament }\end{array}$ \\
\hline Knee 1 & 1,7 & 6,4 & 1,3 & 3,3 \\
\hline Knee 2 & 1,8 & 4 & 1,8 & 2,7 \\
\hline Knee 3 & 2,1 & 5,3 & 0,7 & 3,1 \\
\hline Mean value & 1,86 & 5,23 & 1,26 & 3,03 \\
\hline
\end{tabular}

\section{DISCUSSION}

ACL reconstruction is one of the commonest surgical procedures in orthopedic practice. The data suggest that more than 100,000 reconstructions are performed annually in the United States ${ }^{(4,21)}$. However, there is little documentation of vascular complications in the literature. Now that there is greater interest in horizontalization of the femoral tunnel, in an attempt to reproduce the anatomical footprint of the ACL in the femur, it is believed that there is a greater risk of injury to the lateral prime structures of the knee during the surgical procedure.

Vertical positioning by means of a transtibial tunnel has been widely used around the world, with high rates of good and excellent clinical results described in the literature ${ }^{(25-28)}$. However, older studies did not evaluate the rotational stability of these patients, and a portion of these patients continued to present symptoms of instability and positive results in pivot-shift maneuvers ${ }^{(17,29)}$. On the other hand, positioning the neoligament more horizontally in the lateral wall of the lateral femoral condyle, i.e. closer to the anatomical footprint, provides greater rotational stability that adds to the anteroposterior stability, thereby improving postoperative results that continue to present some degree of instability ${ }^{(7,15-18)}$.

Since this technique has gained greater prominence over recent years, further studies comparing the two techniques over the long term are necessary in order 
to define the benefits and possible complications that are not observed with the first technique. Because of the proximity of prime structures at the exit point of the guidewire, and depending on the graft fixation technique for the femoral tunnel, surgeons have taken greater interest in possible lesions involving lateral structures of the femoral condyle, such as the lateral collateral ligament and the lateral superior genicular artery, and their possible complications like osteonecrosis of the lateral femoral condyle and delayed healing of the graft. Concern regarding these issues has led surgeons to choose the new technique in an attempt to improve their postoperative results.

In an anatomical study on cadavers, Neven et al ${ }^{(30)}$ evaluated the risk of injury to lateral and posterolateral structures during drilling of the femoral tunnel in the anatomical position, through a low anteromedial portal with the knee flexed at 120 degrees. They evaluated the distance of the guidewire exit point from the lateral collateral ligament, lateral tendon of the gastrocnemius and popliteal tendon, and concluded that none of these structures was at risk during the procedure. However, the distance from the lateral superior genicular artery was not evaluated, even though this artery is the main source of blood supply to the lateral femoral condyle.

Farrow and Parker ${ }^{(21)}$ evaluated the risk of injury to these structures, except for the genicular artery, in cadavers, and drilled the femoral tunnel by means of knee arthrotomy, using bone structures as the reference points for standardized placement of guidewires with the knee flexed at 90 degrees, in a position that was slightly lateral to the medial femoral condyle and slightly above the anterior cornu of the medial meniscus. They concluded that the femoral tunnel should be drilled with the knee flexed at a minimum of 110 degrees, in order to diminish the risk of injury to lateral structures. In the present study, despite the observed difference between the femoral tunnel and the lateral collateral ligament (depending on the technique used), the risk of ligament injury seemed to be minimized through distances that were considered safe, thus agreeing with data in the literature.

Injury to the lateral superior genicular artery during arthroscopic reconstruction of the ACL is a little-mentioned event, without reports in the literature. On the other hand, osteonecrosis of the lateral femoral condyle has been mentioned by some authors as a complication of this surgical procedure ${ }^{(24,31)}$. We believe that iatrogenic lesions of the lateral superior genicular artery would, despite its rich anastomosing network, be a risk factor for this feared complication. However, little is known regarding the possible consequence of a total vascular lesion in this arterial branch.

The first description of osteonecrosis of the lateral condyle following ACL reconstruction was made by Athanasian et al ${ }^{(24)}$ in 1995 , in a patient with a chronic lesion in the ligament that was reconstructed arthroscopically. Twenty-five months after the surgery, the patient started to present acute pain after suffering an injury in hyperflexion, but without any injury to ligament structures. Magnetic resonance imaging (MRI) demonstrated a defect in the subchondral bone close to the femoral tunnel, in the lateral femoral condyle, along with a chondral lesion in the posterior region of the condyle. In 2010, another description was made by Shenoy et $a l^{(31)}$, in which a patient who had undergone reconstruction using flexor tendons four weeks after knee trauma presented major bone edema on the initial MRI. Fifteen months after the reconstruction, he developed a condition of progressively incapacitating pain that lasted for eight months, with lytic lesions on radiographs and an area of low signal in the lateral condyle.

The rare incidence of necrosis laterally is due to the greater vascularization and the extraosseous anastomotic network present there. The main blood supply for the lateral femoral condyle is provided by the lateral superior genicular artery, but it also consistently receives an anastomotic branch of the lateral inferior genicular artery. This connection is usually present under the lateral collateral ligament and is possibly one of the causes of lower incidence of osteonecrotic events in the lateral condyle ${ }^{(22,23)}$.

Through measurement of the distance between the main branch of this artery and the femoral tunnel, it can be seen that the tunnel and the vessel are close to each other and that there is a high chance of injury to this artery. This suggests that this proximity is the main cause of the rare but existent cases of osteonecrosis of the lateral femoral condyle following ACL reconstruction. Greater proximity while performing the transportal technique makes this a risk resulting from its use.

Another potential risk from using the transportal technique, based on the results from the present study, and resulting from possible lesions of the lateral superior genicular artery, relates to changes to the healing capacity of the neoligament in the femoral tunnel.

The phases of the ligament formation process consist of necrosis, edema, revascularization, fibroblast 
invasion and collagen synthesis, with involvement of the vascularized synovial tissue at the end of this process $^{(32)}$. Ligament formation is taken to be the concept introduced by Amiel et $a l^{(32)}$ in 1986, which consisted of biochemical and histological remodeling of the tendon graft into a ligament.

Several factors may influence ligament formation from grafts, such as isometricity, anatomical positioning, collaboration from the patient, response to healing, biomechanical strength, postoperative rehabilitation and, especially, vascularization ${ }^{(33)}$. Some studies have suggested that the ligament formation process occurs within approximately one year following the surgery.

We believe that injuries to this artery may alter the process of ligament formation from the graft, such that it is delayed and consequently the capacity to reesta-

\section{REFERÊNCIAS}

1. Petersen $W$, Tillmann $B$. [Anatomy and function of the anterior cruciate ligament]. Orthopade. 2002;31(8):710-8.

2. Girgis FG, Marshall JL, Monajem A. The cruciate ligaments of the knee joint. Anatomical, functional and experimental analysis. Clin Orthop Relat Res. 1975;(106):216-31.

3. Petersen W, Zantop T. Anatomy of the anterior cruciate ligament with regard to its two bundles. Clin Orthop Relat Res. 2007;454:35-47.

4. Frank CB, Jackson DW. The science of reconstruction of the anterior cruciateligament. J Bone Joint Surg Am. 1997;79(10):1556-76.

5. Struewer J, Frangen TM, Ishaque B, Bliemel C, Efe T, Ruchholtz S, et al. Knee function and prevalence of osteoarthritis after isolated anterior cruciate ligament reconstruction using bone-patellar tendon-bone graft: long-term follow-up. Int Orthop. 2012;36(1):171-7.

6. Lidén M, Sernert N, Rostgård-Christensen L, Kartus C, Ejerhed L.Osteoarthritic changes after anterior cruciate ligament reconstruction using bone-patellar tendon-bone or hamstring tendon autografts: a retrospective, 7-year radiographic and clinical follow-up study. Arthroscopy. 2008;24(8):899-908.

7. Alentorn-Geli E, Lajara F, Samitier G, Cugat R. The transtibial versus the anteromedial portal technique in the arthroscopic bone-patellar tendon-bone anterior cruciate ligament reconstruction. Knee Surg Sports Traumatol Arthrosc. 2010;18(8):1013-37.

8. Piasecki DP, Bach BR Jr, Espinoza Orias AA, Verma NN. Anterior cruciate ligament reconstruction: can anatomic femoral placement be achieved with a transtibial technique? Am J Sports Med. 2011;39(6):1306-15.

9. Strauss EJ, Barker JU, McGill K, Cole BJ, Bach BR Jr, Verma NN. Can anatomic femoral tunnel placement be achieved using a transtibial technique for hamstring anterior cruciate ligament reconstruction? Am J Sports Med. 2011;39(6):1263-9.

10. Izawa T, Okazaki K, Tashiro Y, Matsubara H, Miura H, Matsuda S, et al. Comparison of rotatory stability after anterior cruciate ligament reconstruction between single-bundle and double-bundle techniques. Am J SportsMed. 2011;39(7):1470-7.

11. Guardamagna L, Seedhom BB, Ostell AE. Double-band reconstruction of the ACL using a synthetic implant: a cadaveric study of knee laxity. J Orthop Sci. 2004;9(4):372-9.

12. Radford WJ, Amis AA, Kempson SA, Stead AC, Camburn M. A comparative study of single- and double-bundle ACL reconstructions in sheep. Knee Surg Sports Traumatol Arthrosc. 1994;2(2):94-9.

13. Hara K, Kubo T, Suginoshita T, Shimizu C, Hirasawa Y. Reconstruction of the anterior cruciate ligament using a double bundle. Arthroscopy. 2000;16(8):860-4.

14. Pastrone A, Ferro A, Bruzzone M, Bonasia DE, Pellegrino P, D’Elicio D, et al. Anterior cruciate ligament reconstruction creating the femoral tunnel through the anteromedial portal. Surgical technique. Curr Ver Musculoskelet Med. 2011;4(2):52-6.

15. Alentorn-Geli E, Samitier G, Alvarez P, Steinbacher G, Cugat R. Anteromedial portal versus transtibial drilling techniques in ACL reconstruction: a blinded crosssectional study at two- to five-year follow-up. Int Orthop. 2010;34(5):747-54.

16. Steiner ME, Battaglia TC, Heming JF, Rand JD, Festa A, Baria M. Independent drilling outperforms conventional transtibial drilling in anterior cruciate ligament reconstruction. Am J Sports Med. 2009;37(10):1912-9

17. Loh JC, Fukuda Y, Tsuda E, Steadman RJ, Fu FH, Woo SL. Knee stability and graft function following anterior cruciate ligament reconstruction: Comparison between 11 o'clock and 10 o'clock femoral tunnel placement. 2002 Richard blish a biologically integrated ligament is also delayed.

This study presents certain limitations. The small number of knees evaluated limits the statistical value of the results. No assessment was made regarding intraosseous vascular trauma, which might have made the results and suppositions from this study even more significant.

\section{CONCLUSION}

Use of the transportal technique for arthroscopic reconstruction of the ACL presents greater likelihood of injury to the lateral genicular artery and the insertion of the lateral collateral ligament, thus favoring postsurgical complications such as knee instability, osteonecrosis of the lateral femoral condyle and ligament formation from the graft.

O'Connor Award paper. Arthroscopy. 2003;19(3):297-304

18. Scopp JM, Jasper LE, Belkoff SM, Moorman CT 3rd. The effect of oblique femoral tunnel placement on rotational constraint of the knee reconstructed using patellar tendon autografts. Arthroscopy. 2004;20(3):294-9.

19. Maak TG, Bedi A, Raphael BS, Citak M, Suero EM, Wickiewicz T, et al. Effect of femoral socket position on graft impingement after anterior cruciate ligament reconstruction. Am J Sports Med. 2011;39(5):1018-23.

20. Farrow LD, Liu RW. Lateral anatomic structures at risk during transepiphyseal anterior cruciate ligament reconstruction. J Knee Surg. 2010;23(4):209-13.

21. Farrow LD, Parker RD. The relationship of lateral anatomic structures to exiting guide pins during femoral tunnel preparation utilizing an accessory medial portal. Knee Surg Sports Traumatol Arthrosc. 2010;18(6):747-53.

22. Lankes M, Petersen W, Hassenpflug J. [Arterial supply of the femoral condyles]. Z Orthop Ihre Grenzgeb. 2000;138(2):174-80.

23. Reddy AS, Frederick RW. Evaluation of the intraosseous and extraosseous blood supply to the distal femoral condyles. Am J Sports Med. 1998;26(3):415-9.

24. Athanasian EA, Wickiewicz TL, Warren RF. Osteonecrosis of the femoral condyle after arthroscopic reconstruction of a cruciate ligament. Report of two cases. J Bone Joint Surg Am. 1995;77(9):1418-22.

25. Shi DL, Wang YB, Ai ZS. Effect of anterior cruciate ligament reconstruction on biomechanical features of knee in level walking: a meta-analysis. Chin Med J (Engl). 2010;123(21):3137-42.

26. Leal-Blanquet J, Alentorn-Geli E, Tuneu J, Valentí JR, Maestro A. Anterior cruciate ligament reconstruction: a multicenter prospective cohort study evaluating 3 different grafts using same bone drilling method. Clin J Sport Med. 2011;21(4):294-300.

27. Weninger P, Zifko B, Liska M, Spitaler R, Pelinka H, Hertz H. Anterior cruciate ligament reconstruction using autografts and double biodegradable femoral cross-pin fixation: functional, radiographic and MRI outcome after 2-year minimum follow-up. Knee Surg Sports Traumatol Arthrosc. 2008;16(11):988-95.

28. Anders JO, Struwe MS, Sander K, Layher F, Venbrocks RA. [Radiological and clinical functional examinations 36 months after anterior cruciate ligament repair by a patellar tendon graft]. Z Orthop Unfall. 2007;145(6):719-25.

29. Lee MC, Seong SC, Lee S, Chang CB, Park YK, Jo H, et al. Vertical femoral tunnel placement results in rotational knee laxity after anterior cruciate ligament reconstruction. Arthroscopy. 2007;23(7):771-8.

30. Neven E, D'Hooghe P, Bellemans J. Double-bundle anterior cruciate ligament reconstruction: a cadaveric study on the posterolateral tunnel position and safety of the lateral structures. Arthroscopy. 2008;24(4):436-40.

31. Shenoy PM, Shetty GM, Kim DH, Wang KH, Choi JY, Nha KW. Osteonecrosis of the lateral femoral condyle following anterior cruciate ligament reconstruction: is bone bruising a risk factor? Arch Orthop Trauma Surg. 2010;130(3):413-6.

32. Amiel D, Kleiner JB, Roux RD, Harwood FL, Akeson WH. The phenomenon of "ligamentization": anterior cruciate ligament reconstruction with autogenous patellar tendon. J Orthop Res. 1986;4(2):162-72.

33. Marumo K, Saito M, Yamagishi T, Fujii K. The "ligamentization" process in human anterior cruciate ligament reconstruction with autogenous patellar and hamstring tendons: a biochemical study. Am J Sports Med. 2005;33(8):1166-73. 